\title{
A ZERO-ONE PROPERTY OF MIXING SEQUENCES OF EVENTS ${ }^{1}$
}

\author{
BY L. SUCHESTON
}

Communicated by J. L. Doob, February 27, 1962

A sequence of events $\left\{A_{n}\right\}_{1}^{\infty}$ is mixing if for each event $M, P\left(A_{n} M\right)$ $-P\left(A_{n}\right) P(M) \rightarrow 0$; if it is also assumed that $P\left(A_{n}\right) \rightarrow \alpha, 0 \leqq \alpha \leqq 1$, $\left\{A_{n}\right\}$ is called mixing with density $\alpha$. A sequence of events $\left\{A_{n}\right\}$ is zero-one if its tail is trivial; semi-zero-one if every subsequence of $\left\{A_{n}\right\}$ admits a zero-one subsequence.

Theorem 1. A sequence of events is mixing if and only if it is semizero-one.

OUtLINE OF PROOF. Denote by $\mathfrak{Q}_{n}, \mathfrak{B}_{n}, \mathfrak{C}_{n}$, respectively, the $\sigma$ fields generated by the event $A_{n}$, by the events $A_{1}, \cdots, A_{n}$, and by the events $A_{n}, A_{n+1}, \cdots ; \mathfrak{e}=\bigcap_{1}^{\infty} \mathbb{C}_{n}$ is the tail of the sequence $\left\{A_{n}\right\}$. If $\left\{A_{n}\right\}$ is zero-one, then for every bounded random variable $X$ $E\left(X / \mathfrak{e}_{n}\right) \rightarrow E(X)$ with probability one and in $L_{1}$ mean. One shows that if $P\left(A_{n}\right) \rightarrow \alpha, 0<\alpha<1$, then $\left\{A_{n}\right\}$ is mixing if and only if, for each bounded random variable $X, E\left(X / \mathfrak{Q}_{n}\right) \rightarrow E(X)$ in $L_{1}$ mean ("in $L_{1}$ mean" may here be replaced by "in probability" or by "uniformly except on a null event"). Hence a zero-one sequence, and also a semizero-one sequence, will be mixing. Now denote by $A^{v}$ the event $A$ or its complement and by $I_{A}$ the characteristic function of $A$. Let $\left\{A_{n}\right\}$ be a sequence such that all events $A_{1}^{0} \cdots A_{n}^{0}, n=1,2, \cdots$, are not null and let $Q$ be the independent probability measure on $\mathfrak{C}_{1}$ with $Q\left(A_{n}\right)=\alpha, n=1,2, \cdots, 0<\alpha<1$. Set

$$
X_{n}=\sum I_{A_{1} \cdots A_{n}^{v}}^{v} \frac{P\left(A_{1}^{v} \cdots A_{n}^{v}\right)}{Q\left(A_{1}^{v} \cdots A_{n}^{v}\right)}, \quad n=1,2, \cdots,
$$

where the summation extends over all events $A_{1}^{0} \cdots A_{n}^{0}$ of $\Theta_{n}$. It is shown that every sequence of events mixing with density $\alpha$ admits a subsequence $\left\{A_{n}\right\}$ such that the $X_{n}$ 's defined by (1) are uniformly integrable with respect to the measure $Q$ (even uniformly bounded by $1-\epsilon, 1+\epsilon$ where $\epsilon$ is arbitrarily small). Doob's discussion [1, pp. $343 \mathrm{ff}$.] shows that $P$ is absolutely continuous with respect to $Q$ on $\mathfrak{e}_{1}$; by Kolmogorov's zero-one law $\left\{A_{n}\right\}$ is $Q$ zero-one, hence $\left\{A_{n}\right\}$ is also $P$ zero-one. It follows that a mixing sequence is semi-zero-one,

1 This work was supported by the National Science Foundation Grant NSF G-14446. 
unless it is mixing with density zero or one in which case one shows the existence of a zero-one subsequence by a direct argument.

From Theorem 1, one obtains that Kolmogorov automorphisms are mixing in all degrees [cf. Rokhlin 2, p. 14]. In probabilistic formulation: discrete-parameter stationary stochastic processes with trivial tail are mixing in all degrees. (The tail of a process $\left\{X_{n}\right\}_{-\infty}^{\infty}$ is the $\sigma$-field $\cap_{-\infty}^{\infty} \mathcal{e}_{n}$ where $\mathcal{C}_{n}$ is generated by $\cdots X_{n-1}, X_{n}$.) Via distribution functions Theorem 1 may also be applied to processes not necessarily stationary. A sequence of random variables $\left\{X_{n}\right\}_{1}^{\infty}$ is called mixing if for some dense set $D$ on the real line $R$ the sequence of events $\left\{A_{n}(y)\right\}$ is mixing for each $y \in D$, where $A_{n}(y)$ is defined on $R$ by

$$
A_{n}(y)=\left[X_{n}<y\right], \quad n=1,2, \cdots .
$$

If $\left\{A_{n}(y)\right\}$ is mixing with density $\bar{F}(y)$ for $y \in D$, then $\bar{F}$ determines a distribution function $F$ defined on $R$ and $P\left(A_{n}(y)\right)$ converges to $F(y)$ on the continuity set of $F(y)$; the sequence of random variables $\left\{X_{n}\right\}$ is then called mixing with the limiting distribution function $F(y)$; this last notion was introduced by Rényi [3]. It follows from Theorem 1 that if a sequence of random variables $\left\{X_{n}\right\}$ is semi-zeroone, i.e. if every subsequence contains a subsequence with trivial tail, then $\left\{X_{n}\right\}$ is mixing. It is further shown under rather weak assumptions that mixing is invariant under change of measure. A probability measure $Q$ is semicontinuous with respect to $P$ on a sequence of random variables $\left\{X_{n}\right\}$ if every subsequence of $\left\{X_{n}\right\}$ contains a further subsequence $\left\{Y_{n}\right\}$ such that $Q$ is absolutely continuous with respect to $P$ on the tail of $\left\{Y_{n}\right\}$.

THEOREM 2. Let a sequence of random variables $\left\{X_{n}\right\}$ be $P$ mixing (with a limiting distribution function $F(y)$ ). If $Q$ is a probability measure semicontinuous with respect to $P$ on $\left\{X_{n}\right\}$, then the sequence $\left\{X_{n}\right\}$ is $Q$ mixing (with the limiting distribution function $F(y)$ ).

In the proof, the invariance of mixing is obtained from Theorem 1 while the invariance of the limiting distribution is derived from the second theorem of Andersen and Jessen [4].

Theorem 2 extends Theorem 2 of Abbot and Blum [5] and certain results on invariance of limiting distributions of Rényi and Révész. Namely in Theorem 4 of Rényi [3] concerned with sums of independent random variables and in Examples 3 and 4 of Rényi and Révész [6] concerned with certain Markov chains, the premises may be weakened by assuming semicontinuity of $Q$ with respect to $P$ on the studied sequences of averages of random variables, instead 
of absolute continuity of $Q$ with respect to $P$ on $Q$; the conclusions may be strengthened by asserting $Q$ mixing of these sequences with the limiting distribution function $F(y)$, instead of only the convergence of the distribution functions of the averages to $F(y)$.

\title{
BIBLIOGRAPHY
}

1. J. L. Doob, Stochastic processes, Wiley, New York, 1953.

2. V. A. Rokhlin, New progress in the theory of transformations with invariant measure, Uspehi Mat. Nauk (1960), English translation published by the London Math. Soc.

3. A. Rényi, On mixing sequences of sets, Acta Math. Acad. Sci. Hungar. 9 (1958), 215-228.

4. E. S. Andersen and B. Jessen, Some limit theorems on set-functions, Danske Vid. Selsk. Mat.-Fys. Medd. 25 (1948).

5. J. H. Abbott and R. J. Blum, On a theorem of Rényi concerning mixing sequences of sets, Ann. Math. Statist. 32 (1961), 257-260.

6. A. Rényi and P. Révész, On mixing sequences of random variables, Acta Math. Acad. Sci. Hungar. 9 (1958), 389-393.

University of Wisconsin, Milwaukee

\section{THE EQUATION $\left(\partial^{2} / \partial x^{2}+\partial^{2} / \partial y^{2}+\left(x^{2}+y^{2}\right)(\partial / \partial t)\right)^{2} u+\partial^{2} u / \partial t^{2}=f$, WITH REAL COEFFICIENTS, IS "WITHOUT SOLUTIONS"}

\author{
BY FRANÇOIS TREVES ${ }^{1}$ \\ Communicated by Lipman Bers, February 20, 1962
}

Indeed, the equation can be written $P P^{*}\left(P P^{*}\right)^{*} u=f$, where $P$ is Lewy's operator $\partial / \partial \bar{z}+i z(\partial / \partial t),{ }^{2} z=x+i y$, and the star operation replaces the coefficients of a differential operator by their complex conjugates. Hörmander has shown ${ }^{3}$ that, whatever be the open set $\Omega$, there is a function $f \in C_{0}^{\infty}(\Omega)$ such that the equation $P v=f$ does not have any distribution solution $v \in D^{\prime}(\Omega)$.

YESHIVA UNIVERSITY

${ }^{1}$ Sloan fellow. Yeshiva University, New York City.

${ }^{2} \mathrm{H}$. Lewy, An example of a smooth linear partial differential equation without solution, Ann. of Math. (2) 66 (1957), 155.

${ }^{3}$ L. Hörmander, Differential equations without solutions, Math. Ann. 140 (1960), 169. 